\title{
A NEW PROOF OF THE FUNCTIONAL EQUATION OF DIRICHLET $L$-FUNCTIONS ${ }^{1}$
}

\author{
BRUCE C. BERNDT
}

ABSTRACT. A simple proof, using contour integration, of the functional equation of Dirichlet $L$-functions is given.

Let $\chi$ be a nonprincipal, primitive character modulo $k$. Let

$$
G(z, \chi)=\sum_{j=1}^{k-1} \chi(j) e^{2 \pi i j z / k}
$$

denote a Gaussian sum, and put $G(\chi)=G(1, \chi)$. We shall need two fundamental properties of Gaussian sums. If $n$ is an integer [1, p. 312],

$$
G(n, \bar{\chi})=\chi(n) G(\bar{\chi}) .
$$

Secondly $[1$, p. 313],

$$
G(\chi) G(\bar{\chi})=\chi(-1) k .
$$

THEOREM. The Dirichlet L-function,

$$
L(s, \chi)=\sum_{n=1}^{\infty} \chi(n) n^{-s}, \quad \sigma=\operatorname{Re} s>0,
$$

can be analytically continued to an entire function which satisfies the functional equation

$$
L(1-s, \chi)=(k / 2 \pi)^{s} k^{-1} G(\chi) \Gamma(s) L(s, \bar{\chi})\left\{e^{-\pi i s / 2}+\chi(-1) e^{\pi i s / 2}\right\} .
$$

Proof. For $\sigma>1$, it is quite easy to show that [2, pp. 194, 200]

$$
\Gamma(s) L(s, \chi)=\int_{0}^{\infty} \frac{x^{s-1} G(i k x / 2 \pi, \chi) d x}{1-e^{-k x}} .
$$

Equation (4) is the starting point for a proof of (3) by Ayoub [2], but otherwise our proof has nothing in common with his.

Received by the editors July 9, 1971.

AMS (MOS) subject classifications (1969). Primary 1041; Secondary 1040, 1043.

$K e y$ words and phrases. Dirichlet $L$-function, functional equation, primitive character, Gaussian sum.

${ }^{1}$ Research supported by NSF grant GP 21335.

(c) American Mathematical Society 1973 
Assume that $s$ is real and $s>1$. If $m$ is a positive integer, let $C_{m}$ denote the positively oriented, closed contour consisting of $\Gamma_{m}$, the right half of the circle with center $(0,0)$ and radius $m+\frac{1}{2}$, together with the vertical diameter indented at the origin by a semicircle $\Gamma_{\varepsilon}$ of radius $\varepsilon<1$ in the right half plane. Define

$$
F(z)=\pi e^{-\pi i z} G(z, \bar{\chi}) / G(\bar{\chi}) z^{s} \sin (\pi z),
$$

where $z^{s}$ is given its principal value. On the interior of $C_{m}, F$ is analytic except for simple poles at $z=1, \cdots, m$. The residue of $F$ at the positive integer $n$ is $G(n, \bar{\chi}) / G(\bar{\chi}) n^{s}=\chi(n) n^{-s}$, upon the use of (1). Hence, by the residue theorem,

$$
\frac{1}{2 \pi i} \int_{C_{m}} F(z) d z=\sum_{n=1}^{m} \chi(n) n^{-s} .
$$

Now, $\left|e^{-\pi i z} G(z, \bar{\chi}) / \sin (\pi z)\right|$ has period $k$ and tends to zero exponentially as $\operatorname{Im} z$ tends to $\pm \infty$. Thus, there exists a positive number $M$, independent of $m$, such that for all $z$ on $\Gamma_{m}$,

$$
\left|e^{-\pi i z} G(z, \bar{\chi}) / \sin (\pi z)\right| \leqq M
$$

Since $s>1$, clearly the integral of $F$ over $\Gamma_{m}$ tends to 0 as $m$ tends to $\infty$. Hence, upon letting $m$ tend to $\infty$ in (5), we find that

$$
\begin{aligned}
L(s, \chi)= & \int_{i \varepsilon}^{i \infty} \frac{G(z, \bar{\chi}) d z}{G(\bar{\chi}) z^{s}\left(1-e^{2 \pi i z}\right)}+\int_{-i \varepsilon}^{-i \infty} \frac{e^{-2 \pi i z} G(z, \bar{\chi}) d z}{G(\bar{\chi}) z^{s}\left(1-e^{-2 \pi i z}\right)} \\
& +\frac{1}{2 \pi i} \int_{\Gamma_{\varepsilon}} F(z) d z .
\end{aligned}
$$

The two infinite integrals on the right side of (6) each converge uniformly on any compact set of the complex $s$-plane. Thus, (6) shows that $L(s, \chi)$ can be analytically continued to an entire function of $s$, and (6) is then valid for all $s$.

Now suppose that $s<0$. Since $G(0, \chi)=0$, it is trivial to see that the integral over $\Gamma_{\varepsilon}$ on the right side of (6) tends to 0 as $\varepsilon$ tends to 0 . Letting $\varepsilon$ tend to 0 in (6), we then obtain for $s<0$,

$$
\begin{aligned}
L(s, \chi)= & i e^{-\pi i s / 2} \int_{0}^{\infty} \frac{G(i y, \bar{\chi}) d y}{G(\bar{\chi}) y^{s}\left(1-e^{-2 \pi y}\right)}-i e^{\pi i s / 2} \int_{0}^{\infty} \frac{e^{-2 \pi y} G(-i y, \bar{\chi}) d y}{G(\bar{\chi}) y^{s}\left(1-e^{-2 \pi y}\right)} \\
= & i e^{-\pi i s / 2}(k / 2 \pi)^{1-s} \int_{0}^{\infty} \frac{G(i k y / 2 \pi, \bar{\chi}) d y}{G(\bar{\chi}) y^{s}\left(1-e^{-k y}\right)} \\
& -i e^{\pi i s / 2}(k / 2 \pi)^{1-s} \int_{0}^{\infty} \frac{e^{-k y} G(-i k y / 2 \pi, \bar{\chi}) d y}{G(\bar{\chi}) y^{s}\left(1-e^{-k y}\right)}
\end{aligned}
$$


If in the definition of $G(z, \chi)$ we replace $j$ by $k-j$, we find that

$$
e^{-k y} G(-i k y / 2 \pi, \bar{\chi})=\chi(-1) G(i k y / 2 \pi, \bar{\chi}) \text {. }
$$

Hence, with the use of (4), (7) reduces to

(8) $L(s, \chi)=i(k / 2 \pi)^{1-s} \Gamma(1-s) L(1-s, \bar{\chi})\left\{e^{-\pi i s / 2}-\chi(-1) e^{\pi i s / 2}\right\} / G(\bar{\chi})$.

If we replace $s$ by $1-s$, apply (2), and use analytic continuation, (8) reduces to (3), and the proof is complete.

The author is grateful to members of the Number Theory Seminar at the University of Illinois for suggesting a few simplifications in the author's original proof.

\section{REFERENCES}

1. Raymond Ayoub, An introduction to the analytic theory of numbers, Math. Surveys, no. 10, Amer. Math. Soc., Providence, R.I., 1963. MR 28 \#3954.

2. —, On L-functions, Monatsh. Math. 71 (1967), 193-202. MR 35 \#5401.

Department of Mathematics, University of Illinois, Urbana, Illinois 61801 